http://dx.doi.org/10.17776/csj.16647

\title{
Upper and Lower Soft Contra Continuous Multifunctions
}

\author{
Metin AKDA $\breve{G}^{*}$ \\ Cumhuriyet University, Institute of Science, Department of Chemistry, 58140 Sivas, Turkey
}

Received: 10.03.2016; Accepted: 06.04.2016

\begin{abstract}
In this paper, we introduce and study upper (lower) soft contra continuous multifunctions. Some characterizations and several properties concerning upper (lower) soft contra continuous multifunctions are obtained..The relationships between upper (lower) soft continuous multifunction and some known concepts are also discussed.
\end{abstract}

Keywords: Multifunction, contra-continuous multifunction, soft continuous multifunction, soft topological spaces.

\section{Esnek Küme Değerli Fonksiyonların Alttan ve Üstten \\ Contra Süreklilikleri}

Özet. Bu makalede, öncelikle esnek küme değerli dönüşümlerin alttan ve üstten kontra sürekliliği tanımlanmıştır. Sonra bu sürekliliğin bazı karakterizasyonları verilerek çeşitli özellikleri incelenmiştir. Sonunda ise esnek sürekli küme değerli dönüşüm ile arasındaki ilişki araştırılmıştır.

Anahtar Kelimeler: Küme-değerli fonksiyon, contra-sürekli küme-değerli fonksiyon, esnek süreklilik, esnek küme düğerli fonksiyon, esnek topolojik uzay.

\section{INTRODUCTION}

Molodtsov [1] introduced the concept of soft sets. Soft set theory has rich potential for practical applications in several sciences such as economics, engineering, environmental science, social science, medical science etc. Pei and Miao [2] investigated the relationships between soft sets and information systems. Ça ğ man et al [5] defined a soft topological space. Zorlutuna et al. [8] studied some concepts in soft topological spaces. Ça $\breve{g}$ man and et al. redefined the operations of the soft sets and constructed a uni-int decision making method by using these new operations [7]. Then Akda $\breve{g}$ and Erol $[3,11,12]$ introduced the concept of soft multifunction and studied their properties. Many researcher studied on soft set theory $[6,9,10]$ etc. One of the important and basic topics in the theory of classical point set topology and several branches of mathematics, which have been researched by many authors, is continuity of functions. This concept has been extended to the setting of multifunctions. In this paper we study to the concepts of soft upper and soft lower contra- continuous soft multifunctions and obtain some characterizations of such multifunctions.

\section{PRELIMINIARIES AND BASIC PROPERTIES}

Definition 1 [1] Let $X$ be an initial universe and $E$ be a set of parameters. Let $P(X)$ denote the power set of $X$ and $A$ be a non-empty subset of $E$. A pair $(F, A)$ is called a soft set over $X$, where $F$ is a mapping given by $F: A \rightarrow P(X)$. In other words, a soft set over $X$ is a parameterized family of subsets of the universe $X$. For $e \in A, F(e)$ may be considered as the set of $e$-approximate elements of the soft set $(F, A)$.

\footnotetext{
* Corresponding author. Email address: metinakdag0@ gmail.com 
Definition 2 [7] A soft set $(F, A)$ over $X$ is called a null soft set, denoted by $\Phi_{A}$, if $F(e)=\varnothing$ for all $e \in A$. If $A=E$, then the null soft set is called universal null soft set, denoted by $\Phi$.

Definition 3 [7] A soft set $(F, A)$ over $X$ is called an absolute soft set, denoted by $\tilde{A}$, if $F(e)=X$ for all $e \in A$. If $A=E$, then the absolute soft set is called universal soft set, denoted by $\tilde{X}$.

Definition 4 4] Let $Y$ be a non-empty subset of $X$, then $Y$ denotes the soft set $(Y, E)$ over $X$ for which $Y(e)=Y$, for all $e \in E$.

Definition 5 [7] The union of two soft sets of $(F, A)$ and $(G, B)$ over the common universe $X$ is the soft set $(H, C)$, where $C=A \cup B$ and for all $e \in C$,

$$
H(e)= \begin{cases}F(e), & \text { if } e \in A-B \\ G(e), & \text { if } e \in B-A \\ F(e) \cup G(e), & \text { if } e \in A \cap B\end{cases}
$$

We write $(F, A) \bar{\cup}(G, B)=(H, C)$.

Definition 6 [7] The intersection $(H, C)$ of two soft sets $(F, A)$ and $(G, B)$ over a common universe $X$, denoted $(F, A) \bar{\cap}(G, B)$, is defined as $C=A \cap B$ and $H(e)=F(e) \cap G(e)$ for all $e \in C$.

Definition 7 [7] Let $(F, A)$ and $(G, B)$ be two soft sets over a common universe $X$. $(F, A) \bar{\complement}(G, B)$ , if $A \subset B$ and $F(e) \subset G(e)$ for all $e \in A$.

Definition 8 [7] For a soft set $(F, A)$ over $X$ the relative complement of $(F, A)$ is denoted by $(F, A)^{c}$ and is defined by $(F, A)^{c}=\left(F^{c}, A\right)$, where $F^{c}: A \rightarrow P(X)$ is a mapping given by $F^{c}(\alpha)=X-F(\alpha)$ for all $\alpha \in A$.

Proposition 1 [10] Let $(G, A),(H, A),(S, A),(T, A)$ be soft sets in $X$. Then the following statements are true;

i) If $(G, A) \bar{\cap}(H, A)=\Phi$, then $(G, A) \bar{\subset}(H, A)^{c}$

ii) $(G, A) \bar{\cup}(G, A)^{c}=\tilde{X}$

iii) If $(G, A) \bar{\subset}(H, A)$ and $(H, A) \bar{\subset}(S, A)$, then $(G, A) \bar{\subset}(S, A)$

iv) If $(G, A) \bar{\subset}(H, A)$ and $(S, A) \bar{\subset}(T, A)$ then $(G, A) \bar{\cap}(S, A) \bar{\subset}(H, A) \bar{\cap}(T, A)$

v) $(G, A) \bar{\subset}(H, A)$ if and only if $(H, A)^{c} \bar{\subset}(G, A)^{c}$. 
Definition 9 [11] The soft set $(G, E)$ over $X$ is called a soft point in $X$, denoted by $E_{e}^{\chi}$, if for $e \in E$ there exist $x \in X$ such that $G(e)=\{x\}$ and $G\left(e^{\prime}\right)=\varnothing$ for all $e^{\prime} \in E-\{e\}$.

Definition 10 [11] The soft point $E_{e}^{\chi}$ is said to be in the soft set $(H, E)$, denoted by $E_{e}^{\chi} \in(H, E)$, if $\chi \in H(e)$.

Proposition 2 [10] Let $E_{e}^{\chi}$ be a soft point and $(H, E)$ be a soft set in $X$. If $E_{e}^{\chi \bar{\epsilon}}(H, E)$, then $E_{e}^{\chi} \bar{\ddagger}(H, E)^{c}$.

Definition 11 [4] Let $\tau$ be the collection of soft sets over $X$, then $\tau$ is said to be a soft topology on $X$ if satisfies the following axioms.

(1) $\Phi, X$ belong to $\tau$,

(2) the union of any number of soft sets in $\tau$ belongs to $\tau$,

(3) the intersection of any two soft sets in $\tau$ belongs to $\tau$.

The triplet $(X, \tau, E)$ is called a soft topological space over $X$. Let $(X, \tau, E)$ be a soft topological space over $X$, then the members of $\tau$ are said to be soft open sets in $X$. A soft set $(F, A)$ over $X$ is said to be a soft closed set in $X$, if its relative complement $(F, A)^{c}$ belongs to $\tau$.

Definition 12 [11] Let $S(X, E)$ and $S(Y, K)$ be two soft classes. Let $u: X \rightarrow Y$ be multifunction and $p: E \rightarrow K$ be mapping. Then a soft multifunction $F: S(X, E) \rightarrow S(Y, K)$ is defined as follows:

For a soft set $(G, E)$ in $S(X, E),(F(G, E), K)$ is a soft set in $(Y, K)$ given by

$$
F(G)(k)= \begin{cases}p-1^{\cup u(G(e))}(k) \cap E \neq \varnothing^{\prime} & p^{-1}(k) \cap E \neq \varnothing \\ \varnothing, & \text { otherwise }\end{cases}
$$

for $k \in K .(F(G, E), K)$ is called a soft image of a soft set $(G, E)$.

Moreover, $F(G, E)=\bar{\cup}\left\{F\left(E_{e}^{\chi}\right): E_{e}^{\chi} \bar{\in}(G, E)\right\}$ for a soft subset $(G, E)$ of $X$.

Definition 13 [11] Let $F: S(X, E) \rightarrow S(Y, K)$ be a soft multifunction. The soft upper (lower) inverse image of $(H, K)$ denoted by $F^{+}(H, K)$ and $F^{-}(H, K)$ respectively defined as follows;

$$
\begin{aligned}
& F^{+}(H, K)=\left\{E_{e}^{\chi} \bar{\in} \tilde{X}: F\left(E_{e}^{\chi}\right) \bar{\subset}(H, K)\right\} \\
& F^{-}(H, K)=\left\{E_{e}^{\chi} \bar{\in} \tilde{X}: F\left(E_{e}^{\chi}\right) \bar{\cap}(H, K) \neq \Phi\right\} . \text { Also, } F(\tilde{X})=\cup F\left(E_{e}^{\chi}\right) .
\end{aligned}
$$

Definition 14 [12] Let $F, G: X \rightarrow Y$ be two soft multifunctions. For $E_{e}^{\chi} \in \tilde{X}$, the combination and intersection of $F$ and $G$ is denoted by 


$$
\begin{aligned}
& (F \cup G)\left(E_{e}^{\chi}\right)=F\left(E_{e}^{\chi}\right) \cup G\left(E_{e}^{\chi}\right), \\
& (F \cap G)\left(E_{e}^{\chi}\right)=F\left(E_{e}^{\chi}\right) \cap G\left(E_{e}^{\chi}\right) .
\end{aligned}
$$

Definition 15 [11] Let $F: S(X, E) \rightarrow S(Y, K)$ and $G: S(X, E) \rightarrow S(Y, K)$ be two soft multifunctions. Then, $F$ equal to $G$ if $F\left(E_{e}^{\chi}\right)=G\left(E_{e}^{\chi}\right)$ for each $E_{e}^{\chi} \in X$.

Definition 16 [11] The soft multifunction $F: S(X, E) \rightarrow S(Y, K)$ is called surjective if $p$ and $u$ are surjective.

Theorem 1 [11] Let $F: S(X, E) \rightarrow S(Y, K)$ be a soft multifunction. Then, for soft sets $(F, E),(G, E)$ and for a family of soft sets $\left(G_{i}, E\right)_{i \in I}$ in the soft class $S(X, E)$ the following are hold:
(a) $F(\Phi)=\Phi$
(b) $F(\tilde{X}) \bar{\subset} \tilde{Y}$
(c) $F((G, A) \bar{\cup}(H, B))=F(G, A) \overline{\mathrm{U}} F(H, B)$ in general $F\left(\overline{\mathrm{U}}_{i}\left(G_{i}, E\right)\right)=\overline{\mathrm{U}}_{i} F\left(G_{i}, E\right)$
(d) $F((G, A) \bar{\cap}(H, B)) \bar{\subset} F(G, A) \bar{\cap} F(H, B)$ in general $F\left(\bigcap_{i}\left(G_{i}, E\right)\right) \bar{\subset} \bar{\cap}_{i} F\left(G_{i}, E\right)$
(e) If $(G, E) \bar{\subset}(H, E)$, then $F(G, E) \bar{\subset} F(H, E)$.

Theorem 2 [11] Let $F: S(X, E) \rightarrow S(Y, K)$ be a soft multifunction. Then the follows are true:
(a) $F^{-}(\Phi)=\Phi$ and $F^{+}(\Phi)=\Phi$
(b) $F^{-}(\tilde{Y})=\tilde{X}$ and $F^{+}(\tilde{Y})=\tilde{X}$
(c) $F^{-}((G, K) \bar{\cup}(H, K))=F^{-}(G, K) \overline{\mathrm{U}} F^{-}(H, K)$
(d) $F^{+}(G, K) \overline{\mathrm{U}} F^{+}(H, K) \bar{\subset} F^{+}((G, K) \bar{\cup}(H, K))$
(e) $F^{-}((G, K) \bar{\cap}(H, K)) \bar{\subset} F^{-}(G, K) \bar{\cap} F^{-}(H, K)$
(f] $F^{+}(G, K) \bar{\cap} F^{+}(H, K)=F^{+}((G, K) \bar{\cap}(H, K))$
(g) If $(G, K) \bar{\subset}(H, K)$, then $F^{-}(G, K) \bar{\subset} F^{-}(H, K)$ and $F^{+}(G, K) \bar{\subset} F^{+}(H, K)$.

Proposition 3 [11] Let $F: S(X, E) \rightarrow S(Y, K)$ be a soft multifunction. Then the follows are true:

(a) $(G, A) \bar{\subset} F^{+}(F(G, A)) \bar{\subset} F^{-}(F(G, A))$ for a soft subset $(G, A)$ in $X$. If $F$ is surjectice then $(G, A)=F^{+}(F(G, A))=F^{-}(F(G, A))$ 
(b) $F\left(F^{+}(H, B)\right) \bar{\subset}(H, B) \bar{\subset} F\left(F^{-}(H, B)\right)$ for a soft subset $(H, B)$ in $Y$.

(c) For two soft subsets $(H, B)$ and $(U, C)$ in $Y$ such that $(H, B) \bar{\cap}(U, C)=\Phi$ then $F^{+}(H, B) \bar{\cap} F^{-}(U, C)=\Phi$.

Definition 17 [11] For a multifunction $F_{1}:(X, \tau, E) \rightarrow(Y, \theta, K)$ and a soft multifunction $F_{2}:(Y, \theta, K) \rightarrow(Z, \sigma, L)$, the soft soft multifunction $F_{2} o F_{1}:(X, \tau, E) \rightarrow(Z, \sigma, L)$, is defined by $\left(F_{2} o F_{1}\right)\left(E_{e}^{\chi}\right)=F_{2}\left(F_{1}\left(E_{e}^{\chi}\right)\right)$ for $E_{e}^{\chi} \in X$.

Proposition 4 [11] Let $F:(X, \tau, E) \rightarrow(Y, \sigma, K)$ and $G:(Y, \sigma, K) \rightarrow(Z, \eta, L)$ be two soft multifunction. Then the follows are true:

(a) $\left(F^{-}\right)^{-}=F$

(b) For a soft subset $(T, C)$ in $Z,(G o F)^{-}(T, C)=F^{-}\left(G^{-}(T, C)\right) \quad$ and $(G o F)^{+}(T, C)=F^{+}\left(G^{+}(T, C)\right)$.

Proposition 5 [12] Let $F, G: X \rightarrow Y$ be two soft multifunctions. For a soft set $(H, K)$ in $Y$ the following statements are hold:

(i) $(F \cup G)^{-}(H, K)=F^{-}(H, K) \cup G^{-}(H, K)$.

(ii) $(F \cup G)^{+}(H, K)=F^{+}(H, K) \cup G^{+}(H, K)$

(iii) $(F \cap G)^{-}(H, K) \subset F^{-}(H, K) \cap G^{-}(H, K)$

(iv) $F^{+}(H, K) \cap G^{+}(H, K) \subset(F \cap G)^{+}(H, K)$.

Proposition 6 [12] Let $F: X \rightarrow Y$ be a soft multifunction. Then the following are hold: (i) $F((G, E) \bar{\cup}(H, E))=F(G, E) \overline{\mathrm{U}} F(H, E)$

(ii) $F((G, E) \bar{\cap}(H, E)) \bar{\subset} F(G, E) \bar{\cap} F(H, E)$

(iii) $F(\tilde{X})-F(G, E) \bar{\subset} F(\tilde{X}-(G, E))$.

Proposition 7 [11] Let $(G, K)$ be a soft set over $Y$. Then the followings are true for a soft multifunction $F:(X, \tau, E) \rightarrow(Y, \sigma, K)$ :

(a) $F^{+}(\tilde{Y}-(G, K))=\tilde{X}-F^{-}(G, K)$

(b) $F^{-}(\tilde{Y}-(G, K))=\tilde{X}-F^{+}(G, K)$. 


\section{UPPER AND LOWER SOFT CONTRA-CONTINUOUS MULTIFUNCTIONS}

Definition 18 A soft multifunction $F:(X, \tau, E) \rightarrow(Y, \theta, K)$ is called;

i) soft lower contra-continuous multifunction if for any soft closed set $(H, K)$ in $Y$ with $F\left(E_{e}^{\chi}\right) \bar{\cap}(H, K) \neq \phi$, there exists a soft open set $(B, E)$ in $X$ containing $E_{e}^{\chi}$ such that $F(B, E) \bar{\cap}(H, K) \neq \Phi$.

ii) soft upper contra-continuous multifunction if for any soft closed set $(H, K)$ in $Y$ with $F\left(E_{e}^{\chi}\right) \bar{\subset}(H, K)$, there exists a soft open set $(B, E)$ in $X$ containing $E_{e}^{\chi}$ such that

$F(B, E) \bar{\subset}(H, K)$.

Theorem 3 The following statements are equivalent for a soft multifunction $F:(X, \tau, E) \rightarrow(Y, \theta, K)$.

i) $F$ is soft upper contra-continuous,

ii) $F^{+}(M, K)$ is soft open for any soft closed set $(M, K)$ in $Y$,

iii) $F^{-}(G, K)$ is soft closed for any soft open set $(G, K)$ in $Y$.

Proof. i ) => ii ) Let $(M, K)$ be any soft closed set in $Y$ and $F\left(E_{e}^{\chi}\right) \bar{\subset}(M, K)$ namely $E_{e}^{\chi} \bar{\in} F^{+}(M, K)$. By i ), there exists a soft open set $(A, E)$ containing $E_{e}^{\chi}$ such that $F(A, E) \bar{\subset}(M, K)$. Thus, $E_{e}^{\chi} \bar{\in} \operatorname{In} T\left(F^{+}(M, K)\right)$ and hence $F^{+}(M, K)$ is a soft open set in X.

ii $)=>$ iii $)$ Let $(G, K)$ be a soft open set in $Y$. Then $\tilde{Y}-(G, K)$ is a soft closed set in $Y$. By ii), $F^{+}(Y-(G, K))$ is soft open set. Since $F^{+}(Y-(G, K))=X-F^{-}(G, K)$, then $F^{-}(G, K)$ is soft closed set in $X$.

iii $)=>$ ii $)$ Let $(M, K)$ be a soft closed set in Y. Then $\tilde{Y}-(M, K)$ is a soft open set in $Y$. By iii), $F^{+}(Y-(M, K))$ is soft closed set. Since $F^{+}(\tilde{Y}-(M, K))=\tilde{X}-F^{-}(M, K)$, then $F^{-}(M, K)$ is soft open set in $X$.

ii $)=>$ i) Let $(H, K)$ be any soft closed set in $Y$ and $F\left(E_{e}^{\chi}\right) \bar{\subset}(H, K)$. By (ii), $F^{+}(H, K)$ is soft open set in $X$. Take $(B, E)=F^{+}(H, K)$. Then, $(B, E) \bar{\subset} F^{+}(H, K)$. Thus, $F$ is soft upper contra-continuous.

Definition 19 The soft kernel of a soft set $(H, E)$ in a soft topological space $(X, \tau, E)$ is denoted by $\operatorname{Ker}(H, E)$ and defined as $\operatorname{Ker}(H, E)=\bar{\cap}\{(G, E) \in \tau:(H, E) \bar{\subset}(G, E)\}$. 
Lemma 1 For a soft set $(G, E)$ in a soft topological space $(X, \tau, E)$, if $(G, E) \in \tau$, then $(G, E)=\operatorname{Ker}(G, E)$.

Proof. Obvious from the definition 19.

Theorem 4 Let $F:(X, \tau, E) \rightarrow(Y, \theta, K)$ be a soft multifunction. If

$C l\left(F^{-}(H, K)\right) \bar{\subset} F^{-}(\operatorname{Ker}(H, K))$ for any soft set $(H, K)$ in $Y$, then $F$ is soft upper contracontinuous.

Proof. Suppose that $C l\left(F^{-}(H, K)\right) \bar{\subset} F^{-}(\operatorname{Ker}(H, K))$ for every soft set $(H, K)$ in $Y$. Let $(H, K) \in \theta$. By Lemma $1, C l\left(F^{-}(H, K)\right) \bar{\subset} F^{-}(\operatorname{Ker}(H, K))=F^{-}(H, K)$. This implies that $C l\left(F^{-}(H, K)\right)=F^{-}(H, K)$ and hence $F^{-}(H, K)$ is soft closed set in $X$. Thus, by Theorem 3, $F$ is soft upper contra-continuous.

Definition 20 [11] A soft multifunction $F:(X, \tau, E) \rightarrow(Y, \theta, K)$ is called;

i) soft lower continuous if for any soft open set $(H, K)$ in $Y$ with $F\left(E_{e}^{\chi}\right) \bar{\cap}(H, K) \neq \phi$ there exists a soft open subset $(B, E)$ of $X$ containing $E_{e}^{\chi}$ such that $(B, E) \bar{\subset} F^{-}(H, K)$.

ii) soft upper continuous if for any soft open set $(H, K)$ in $Y$ with $F\left(E_{e}^{\chi}\right) \bar{\complement}(H, K)$ there exists a soft open subset $(B, E)$ of $X$ containing $E_{e}^{\chi}$ such that $(B, E) \bar{\subset} F^{+}(H, K)$.

Remark 1 The notions of soft upper contra-continuous multifunctions and soft upper continuous multifunctions are independent as shown in the following examples.

Example 1 Let $X=\left\{x_{1}, x_{2}\right\}, Y=\left\{y_{1}, y_{2}\right\}, E=\left\{e_{1}, e_{2}\right\}, K=\left\{k_{1}, k_{2}\right\}, \tilde{\tau}=\left\{\Phi, \tilde{X},(G, E)^{c}\right\}$ and $\tilde{v}=\{\Phi, \tilde{Y},(H, K)\}$ where $(G, E)=\left\{\left(e_{2},\left\{x_{1}\right\}\right)\right\}$ and $(H, K)=\left\{\left(k_{1},\left\{y_{1}\right\}\right),\left(k_{2},\left\{y_{2}\right\}\right)\right\}$. Then the multifunction $:(X, \tilde{\tau}, E) \rightarrow(Y, \tilde{v}, K)$ where $u: X \rightarrow Y$ is a multifunction defined by $u\left(x_{1}\right)=\left\{y_{1}\right\}, u\left(x_{2}\right)=Y$ and $p: E \rightarrow K$ is afunction defined by $p\left(e_{1}\right)=k_{2}, p\left(e_{2}\right)=k_{1}$ is soft upper contra-continuous, but it is not soft upper semi-continuous. Because for a soft open set $(H, K)$ in $Y$ $F^{+}(H, K)=(G, E)$ is soft closed set in $X$.

Example 2 Let $X=\left\{x_{1}, x_{2}\right\}, Y=\left\{y_{1}, y_{2}\right\}, E=\left\{e_{1}, e_{2}\right\}, K=\left\{k_{1}, k_{2}\right\}, \tilde{\tau}=\left\{\Phi, \tilde{X},(G, E)^{c}\right\}$ and $\tilde{v}=\{\Phi, \tilde{Y},(H, K)\}$ where $(G, E)=\left\{\left(e_{1},\left\{x_{2}\right\}\right)\left(e_{2}, X\right)\right\}$ and $(H, K)=\left\{\left(k_{1},\left\{y_{1}\right\}\right),\left(k_{2},\left\{y_{2}\right\}\right)\right\}$. Then the multifunction $:(X, \tilde{\tau}, E) \rightarrow(Y, \tilde{v}, K)$ where $u: X \rightarrow Y$ is a multifunction defined by $u\left(x_{1}\right)=\left\{y_{1}\right\}, u\left(x_{2}\right)=Y$ and $p: E \rightarrow K$ is a function defined by $p\left(e_{1}\right)=k_{2}, p\left(e_{2}\right)=k_{1}$ is soft lower contra-continuous, but it is not soft lower semi-continuous. Because for a soft open set $(H, K)$ in $Y \quad F^{-}(H, K)=(G, E)$ is soft closed set in $X$.

Theorem 5 The following statements are equivalent for a soft multifunction $F:(X, \tau, E) \rightarrow(Y, \theta, K):$ 


\section{Upper and Lower Soft Contra Continuous Multifunctions}

i) $F$ is soft lower contra-continuous,

ii) $F^{-}(M, K)$ is soft open for any soft closed set $(M, K)$ in $\mathrm{Y}$,

iii) $F^{+}(G, K)$ is soft closed for any soft open set $(G, K)$ in Y.

Proof. It is similar to that of Theorem 3.

Theorem 6 For a soft multifunction $F:(X, \tau, E) \rightarrow(Y, \theta, K)$, if

$C l\left(F^{+}(H, K)\right) \bar{\subset} F^{+}(\operatorname{Ker}(H, K))$ for every soft set $(H, K)$ in $Y$, then $F$ is soft lower contracontinuous.

Proof. Suppose that $C l\left(F^{+}(H, K)\right) \bar{\subset} F^{+}(\operatorname{Ker}(H, K))$ for every soft set $(H, K)$ in $Y$. Let $(\mathrm{H}, \mathrm{K}) \in \theta$. We have $\quad C l\left(F^{+}(H, K)\right) \bar{\subset} F^{+}(\operatorname{Ker}(H, K))=F^{+}(H, K) \quad$. Thus, $C l\left(F^{+}(H, K)\right)=F^{+}(H, K)$ and hence $F^{+}(H, K)$ is soft closed in $X$. By Theorem 5. $F$ is soft lower contra- continuous.

Definition 21 Let $\left\{F_{i}: X \rightarrow Y: i \in I\right\}$ be a family of soft multifunctions. Then the union $\bigcup_{i \in I} F_{i}$ and the intersection $\mathrm{n}_{i \in I} F_{i}$ defined as follows:

$$
\begin{aligned}
& \mathrm{U}_{i \in I} F_{i}: X \rightarrow Y,\left(\mathrm{U}_{i \in I} F_{i}\right)\left(E_{e}^{\chi}\right)=\mathrm{U}_{i \in I} F_{i}\left(E_{e}^{\chi}\right) \text { and } \\
& \mathrm{n}_{i \in I} F_{i}: X \rightarrow Y,\left(\mathrm{n}_{i \in I} F_{i}\right)\left(E_{e}^{\chi}\right)=\mathrm{n}_{i \in I} F_{i}\left(E_{e}^{\chi}\right) .
\end{aligned}
$$

Theorem 7 If $F_{i}: X \rightarrow Y$ are soft upper contra-continuous multifunctions for $i=1,2, \ldots, n$, then $\cup^{n}{ }_{i=1} F_{i}$ is a soft upper contra-continuous multifunction.

Proof. Let $(H, K)$ be a soft closed set of Y. We will show that $\left(\cup^{n}{ }_{i=1} F_{i}\right)^{+}(H, K)=\left\{E_{e}^{\chi} \in X: \cup_{i=1}^{n} F_{i}\left(E_{e}^{\chi}\right) \bar{\subset}(H, K)\right\} \quad$ is soft open set in $X$. Let $E_{e}^{\chi} \in\left(\cup^{n}{ }_{i=1} F_{i}\right)^{+}(H, K)$. Then $F_{i}\left(E_{e}^{\chi}\right) \bar{\complement}(H, K)$ for $i=1,2 \ldots . . n$. Since $F_{i}: X \rightarrow Y$ is soft upper contra-continuous multifunction for $i=1,2, \ldots, n$, then there exists a soft open set $(U, E)$ containing $E_{e}^{\chi}$ such that $F_{i}\left(E_{e}^{Z}\right) \bar{\subset}(H, K)$ for all $E_{e}^{Z \bar{\epsilon}}(U, E) . \operatorname{LeT}(G, E)=\bigcup_{i=1}^{n}(U, E)$ then $(G, E)$ is soft open set. Then $(G, E) \bar{\subset}\left(\cup^{n}{ }_{i=1} F_{i}\right)^{+}(H, K)$. Thus, $\left(\cup^{n}{ }_{i=1} F_{i}\right)^{+}(H, K)$ is soft open and hence $\cup^{n}{ }_{i=1} F_{i}$ is a soft upper contra-continuous multifunction.

Theorem 8 If $F_{i}: X \rightarrow Y$ are soft lower contra-continuous multifunctions for $i=1,2, \ldots, n$, then $\cup^{n}{ }_{i=1} F_{i}$ is a soft lower contra-continuous multifunction.

Proof. Let $(H, K)$ be a soft closed set of $Y$. We will show that $\left(\cup^{n}{ }_{i=1} F_{i}\right)^{-}(H, K)=$ 


$$
\left\{E_{e}^{\chi} \in X: \cup^{n}{ }_{i=1} F_{i}\left(E_{e}^{\chi}\right) \bar{\cap}(H, K) \neq \phi\right\} \text { is soft open set in } X \text {. Let } E_{e}^{\chi} \in\left(\cup^{n}{ }_{i=1} F_{i}\right)^{-}(H, K) .
$$
Then $F_{i}\left(E_{e}^{\chi}\right) \bar{\cap}(H, K) \neq \Phi$ and hence $F_{i_{o}}\left(E_{e}^{\chi}\right) \bar{\cap}(H, K) \neq \Phi$ for an $i_{0}$. Since $F_{i_{o}}: X \rightarrow Y$ is soft lower contra-continuous multifunction, there exists a soft open set $(U, E)$ containing $E_{e}^{\chi}$ such that $F_{i_{o}}\left(E_{e}^{Z}\right) \bar{\cap}(H, K) \neq \Phi \quad$ for $\quad$ all $\quad E_{e}^{Z} \bar{\in}(U, E)$. Then $\quad \cup^{n}{ }_{i=1} F_{i}\left(E_{e}^{Z}\right) \bar{\cap}(H, K) \neq \phi \quad$ and hence $(U, E) \bar{\subset}\left(\cup^{n}{ }_{i=1} F_{i}\right)^{-}(H, K)$. Thus, $\left(\cup_{i=1}^{n} F_{i}\right)^{-}(H, K)$ is soft open set and hence $\cup^{n}{ }_{i=1} F_{i}$ is soft lower contra-continuous multifunction.

Theorem 9 Let $F:(X, \tau, E) \rightarrow(Y, \theta, K)$ be a soft multifunction and $\left\{\left(U_{i}, E\right): i \in I\right\}$ be a soft open cover for $X$. Then Fis soft lower contra-continuous if and only if $F \mid\left(U_{i}, E\right)$ is a soft lower contra-continuous multifunction for all $i \in I$.

Proof. (=>) Let $E_{e}^{\chi}$ be soft point in $X$ and $(H, K)$ be a soft closed set in $Y$ with $E_{e}^{\chi} \bar{\in} F^{-}(H, K)$. Since $\left\{\left(U_{i}, E\right): i \in I\right\}$ is a soft open cover for $X$, then $E_{e}^{\chi} \bar{\in}\left(U_{i_{o}}, E\right)$ for an $i_{0} \in I$ . We have $F\left(E_{e}^{\chi}\right)=F_{i_{o}}\left(E_{e}^{\chi}\right)$ and hence $E_{e}^{\chi} \bar{\in} F_{i_{o^{-}}}(H, K)$. Since $F \backslash\left(U_{i_{o}}, E\right)$ is soft lower contracontinuous, there exists a soft open set $(B, E)=G \cap\left(U_{i_{o}}, E\right)$ in $U_{i_{o}}$ such that $E_{e}^{\chi} \bar{\in}(B, E)$ and $(B, E)=G \bar{\cap}\left(U_{i_{o}}, E\right) \bar{\subset} F^{-}(H, K) \bar{\cap}\left(U_{i_{o}}, E\right)=F \backslash\left(U_{i_{o}}, E\right)^{-}(H, K)$, where $(B, E)$ is soft open set in $X$. We have $E_{e}^{\chi} \bar{\in}(B, E)=$

$G \bar{\cap}\left(U_{i_{o}}, E\right) \bar{\subset} F \backslash\left(U_{i_{o}}, E\right)^{-}(H, K)=F^{-}(H, K) \bar{\cap}\left(U_{i_{o}}, E\right) \bar{\subset} F^{-}(H, K)$. Hence, $F$ is soft lower contra-continuous.

$(<=)$ Let $E_{e}^{\chi}$ be soft point in $X$ and $E_{e}^{\chi} \bar{\epsilon}\left(U_{i}, E\right)$. Let $(H, K)$ be a soft closed set in $Y$ with $E_{e}^{\chi} \bar{\in} F^{-}(H, K)$. Since $F$ is soft lower contra-continuous and $F\left(E_{e}^{\chi}\right)=F \backslash\left(U_{i_{o}}, E\right)\left(E_{e}^{\chi}\right)$, there exists a soft open set $(U, E)$ containing $E_{e}^{\chi}$ such that $(U, E) \bar{\subset} F^{-}(H, K)$. Take

$(B, E)=\left(U_{i}, E\right) \bar{\cap}(U, E)$. Then $(B, E)$ is soft open set in $\left(U_{i}, E\right)$ containing $E_{e}^{\chi}$. We have $(B, E) \bar{\subset} F^{-}(H, K)$. Thus $F$ is soft lower contra- continuous.

Theorem 10 Let $F:(X, \tau, E) \rightarrow(Y, \theta, K)$ be a soft multifunction and $\left\{\left(U_{i}, E\right): i \in I\right\}$ be a soft open cover for $X$. Then Fis soft upper contra-continuous if and only if $F \mid\left(U_{i}, E\right)$ is a soft upper contra-continuous multifunction for all $i \in I$.

Proof. It is similar to that of Theorem 9. 
Theorem 11 If $F:(X, \tau, E) \rightarrow(Y, \theta, K)$ is an soft upper semi-continuous multifunction and $F_{2}:(Y, \theta, K) \rightarrow(Z, \sigma, L)$ is a soft upper contra-continuous multifunction, then $F_{2} o F_{1}$ is soft upper contra-continuous.

Proof. Let $(M, L)$ be a soft closed set in Z. We have $\left(F_{2} o F_{1}\right)^{+}(M, L)=F_{1}^{+}\left(F_{2}^{+}(M, L)\right)$. Since $F_{2}$ is soft upper contra-continuous, $F_{2}^{+}(M, L)$ is soft open in $Y$. Since $F_{1}$ is upper continuous, $F_{1}^{+}\left(F_{2}^{+}(M, L)\right)=\left(F_{2} o F_{1}\right)^{+}(M, L)$ is soft open in X. Thus, $F_{2} o F_{1}$ is soft upper contra-continuous.

\section{CONCLUSION}

In this paper I study on soft multifunctions which defined between soft topological spaces. I give the definition of upper and lower contra continuous soft multifunctions. Then I obtain characterizations of these soft multifunctions and investigate properties of them. I expect that results in this paper will be basis for further applications of soft multifunctions in soft sets theory.

\section{ACKNOWLEDGEMENTS}

We are grateful the office of scientific research projects of Cumhuriyet University (Project No: AFS-439) for financial supports.

\section{REFERENCES}

[1]. D. Molodtsov, Soft set theory -First results, Comput. Math.with Appl. 37 (4/5) , 19-31, (1999).

[2]. D. Pei and D. Miao, From soft sets to information systems, in Proceedings of the IEEE International Conference on Granular Computing, 2, 617-621 (2005).

[3]. Akdağ, M., Erol F., Upper and Lower Continuity of Soft Multifunctions, Appl. Math. Inf. Sci.,7, 1-8 (2013).

[4]. Shabir M., Naz M., On soft topological spaces, Comput. Math. Appl. 611786-1799 (2011).

[5]. Çağman, N., Karataş S., Enginoğlu S., Soft topology, Computer Math. Appl. 62, 351- 358, (2011).

[6]. Kharal, A., Ahmad, B.: Mappings on soft classes. New Math. Nat. Comput. 7(3), 471- 481 (2011).

[7]. Çağman, N, Enginoglu, S., Soft set theory and uni-int decision making, European Journal of Operational Research, 207 848-855 (2010).

[8]. Zorlutuna, İ., Akdag, M., Min, W.K. and Atmaca, S., Remarks on soft topological spaces, Annals of Fuzzy Mathematics and Informatics, 3, 171-185 (2012).

[9]. Maji, P.K., Biswas, R., Roy, A.R.: Soft set theory. Comput. Math. Appl. 45, 555- 562 (2003).

[10]. Nazmul, S.K. Samanta, S.K., Neighbourhood properties of soft topological spaces, Annals of Fuzzy Mathematics and Informatics (2012).

[11]. Akdağ, M., Erol F., Multifunction Between Soft Topological Spaces, International Journal of Mathematics Trends and Technology-62-69, 20(1) (2015).

[12]. Akdağ, M., Erol F., Hyperspaces of Soft Sets, submitted. 